Roel J.P. Smeets • Jan A.M. Smeitink

Ben A. Semmekrot • Hans R. Scholte

Ronald J.A. Wanders - Lambert P.W.J. van den Heuvel

\title{
A novel splice site mutation in neonatal carnitine palmitoyl transferase II deficiency
}

Received: September 18, 2002 / Accepted: October 29, 2002

\begin{abstract}
Mitochondrial $\beta$-oxidation of long-chain fatty acids requires the concerted action of three tightly integrated membrane-bound enzymes (carnitine palmitoyltransferase I and II and carnitine/acylcarnitine translocase) that transport them into mitochondria. Neonatal onset of carnitine palmitoyltransferase II (CPT II) deficiency is an autosomal recessive, often lethal disorder of this transport. We describe a novel splice-site mutation in the CPT II gene, found in a Moroccan family, of which four out of five children have died from the neonatal form of CPT II deficiency. Mutation detection studies at the mRNA level in the $C P T$ II gene implied that the affected children were homozygous for the previously reported 534T insertion followed by a 25-bp deletion (encompassing bases 534-558). Studies of genomic DNA, however, revealed all patients to be compound heterozygous for this 534Tins/del 25 mutation, and for a new $\mathrm{g} \rightarrow \mathrm{a}$ splice-site mutation in the splice-acceptor site of intron 2. Because of these findings, prenatal diagnosis was performed in chorionic villi of three new pregnancies. This did not reveal new compound heterozygous genotypes, and, after uneventful pregnancies, all children appeared to be healthy. The new mutation is the first splice-site mutation ever identified in CPT II deficiency. The fact that it was not discovered in the patient's cDNA makes this study another example of the incompleteness of mutation detection
\end{abstract}

R.J.P. Smeets · J.A.M. Smeitink $(\varangle)$ - L.P.W.J. van den Heuvel Nijmegen Center for Mitochondrial Disorders, Department of Pediatrics, University Medical Centre Nijmegen, Geert Grooteplein 10, PO BOX 9101, 6500 HB Nijmegen, The Netherlands

Tel. +31-24-3614430; Fax +31-24-3616428

e-mail: J. Smeitink@cukz.umcn.nl

B.A. Semmekrot

Department of Pediatrics, Canisius Wilhelmina Hospital, Nijmegen, The Netherlands

H.R. Scholte

Department of Biochemistry, Erasmus University Medical Centre Rotterdam, Rotterdam, The Netherlands

R.J.A. Wanders

Department of Pediatrics, University of Amsterdam, Academic

Medical Centre, Amsterdam, The Netherlands at the mRNA level in cases where a mutation leads to aberrant splicing or nonsense-mediated messenger decay.

Key words Carnitine palmitoyltransferase II (CPT II) • Mitochondrial $\beta$-oxidation $\cdot$ Molecular analysis $\cdot$ Splice-site mutation $\cdot$ Prenatal diagnosis

\section{Introduction}

Carnitine palmitoyltransferase II (CPT II) deficiency is an autosomal recessive disorder of mitochondrial $\beta$-oxidation of long-chain fatty acids (LCFA). In contrast to mediumchain and short-chain fatty acids, LCFA require a carnitine transport system to enter the mitochondrial matrix where fatty acid $\beta$-oxidation takes place (Bieber 1988; Guzman and Geelen 1993). During this transport, the conversion of long-chain acyl-CoA to acylcarnitine is catalyzed by carnitine palmitoyltransferase I (CPT I), which catalytic and regulatory domains are localized on the outer face of the outer mitochondrial membrane (Fraser et al. 1997). Carnitine/acylcarnitine translocase then shuttles the acylcarnitine through the inner mitochondrial membrane, where it is converted back to an acyl-CoA intermediate by CPT II, an inner mitochondrial membrane enzyme (McGarry et al. 1989).

CPT II deficiency has three distinct clinical forms: a neonatal form, which results in sudden death, and two less severe forms, the early-onset infantile and late-onset adult forms. The lethal neonatal form presents with hypoketotic hypoglycemia and severe hepatomuscular symptoms (Demaugre et al. 1991). The infantile form shows recurrent attacks of acute liver failure with hypoketotic hypoglycemia, resulting in coma and seizures, and transient hepatomegaly. Classical adult-onset CPT II deficiency is clinically characterized by recurrent episodes of muscle pain, muscle weakness, and rhabdomyolysis, triggered by prolonged exercise (DiMauro and DiMauro 1973).

The cloning of the CPT II gene has enabled the identification and analysis of mutations in CPT II patients, as well 
as the correlation of mutant genotypes to clinical phenotypes (Thuillier et al. 2000; Verderio et al. 1995). Truncating CPT II mutations are often associated with the severe neonatal form, while "mild" missense mutations mostly lead to the infantile or adult form. The most common mutation in (adult) CPT II deficiency is the S113L substitution (Taroni et al. 1993), while several other mutations have also been identified in individual patients suffering the late-onset and infantile forms. But to date, only four individual mutations, all located in the CPT II gene, have been found in patients with the neonatal form of CPT II deficiency (Bonnefont et al. 1999).

In this study, we describe a Moroccan family of which four out of five children, born to non-consanguineous parents, died from the neonatal form of CPT II deficiency. Molecular genetic studies were performed to identify disease-causing mutation(s), which would possibly enable prenatal diagnosis.

\section{Materials and methods}

\section{Study participants}

The first CPT II-deficient patient, a girl, was born after an uncomplicated pregnancy of 38 weeks. The second patient, a boy, born after 37 weeks, exhibited intrauterine growth retardation. Shortly before birth, intrauterine cardiac arrhythmias were noticed in this child. The third patient was a premature boy of 35 weeks. The fourth child, a girl, is healthy. The fifth child in this family, a CPT II-deficient boy, was born after an uneventful pregnancy of 37 weeks duration, following labor inducation. All four affected children presented between day one and ten with seizures, lethargy, hypotonia, respiratory insufficiency, cardiac arrhythmias, cardiomegaly, and hepatomegaly. These four patients died due to untreatable cardiorespiratory insufficiency with circulatory failure due to dysrhythmias.

In this study, we used the fifth child as our index patient for molecular analysis, because muscle tissue, blood, and skin fibroblasts from this patient were available.
From all other family members, except from the first child, only DNA (isolated from blood) was available. After establishment of the primary genetic defect, chorionic villi of three new pregnancies were used for prenatal diagnosis.

Biochemical methods

CPT II activity in cultured skin fibroblasts was measured essentially as described by Taroni et al. (1992).

\section{Mutational analyses}

$m R N A$ analysis

Wild-type human cDNA was obtained by reverse transcription of $200 \mathrm{ng}$ poly $\mathrm{A}^{+}$human heart mRNA (Clontech, Woerden, The Netherlands) in $40 \mu$ l total sample volume using Superscript II RNase $\mathrm{H}^{-}$reverse transcriptase, oligo(dT), and random hexamer primers (Life Technologies, Breda, The Netherlands) as described by the manufacturer. Total RNA of the index patient was extracted from cultured human skin fibroblasts (Chomczynski and Sacchi 1987), and $5 \mu \mathrm{g}$ was reverse transcribed as mentioned above. Polymerase chain reaction (PCR) amplification of six overlapping fragments encompassing the complete open reading frame of the human CPT II cDNA was performed according to the protocol for Taq polymerase (Life Technologies). PCR reactions were performed on $1 \mu \mathrm{l}$ cDNA in $25 \mu \mathrm{l}$ total sample volume containing $50 \mathrm{ng}$ of each primer, $0.25 \mathrm{mM}$ dNTPs, $1.5 \mathrm{mM} \mathrm{MgCl}_{2}, 0.5$ units Taq polymerase, and PCR buffer (Life Technologies). PCR fragments were visualized by electrophoresis on a $1 \%$ agarose gel. Direct sequencing of the PCR products was performed using ABI PRISM Dye Terminators on the automated ABI PRISM 377 sequencer according to the manufacturer's protocol (Applied Biosystems, Nieuwekerk a/d IJssel, The Netherlands). All oligonucleotides used for both PCR and direct sequencing were designed according to the human CPT II mRNA sequence published in GenBank (Verderio et al. 1995) (accession number U09648), and are listed in Table 1.

Table 1. Characteristics of primers used for CPT II cDNA polymerase chain reaction (PCR) and sequencing

\begin{tabular}{llll}
\hline Primer name & Sequence $\left(5^{\prime} \rightarrow 3^{\prime}\right)$ & $\mathrm{T}_{\text {an }}\left({ }^{\circ} \mathrm{C}\right)$ & Fragment length (bp) \\
\hline F1 & $\begin{array}{l}\text { cag tgt ctt ggg cgc taa cg } \\
\text { cca ggg tcc cga aat gta gc }\end{array}$ & 52 & 442 \\
R1 & $\begin{array}{l}\text { cat tgg aaa aga act gca tga gc } \\
\text { F2 }\end{array}$ & 58 & 405 \\
R2 & $\begin{array}{l}\text { cga gtt gag ttg aaa agc cg } \\
\text { cgc ttt gtg cct tcc tct ctg }\end{array}$ & 55 & 453 \\
F3 & $\begin{array}{l}\text { cag cat att gtg gga caa gtg g } \\
\text { Rat gag tag tgg caa tga gga gag c }\end{array}$ & 52 & 443 \\
F4 & $\begin{array}{l}\text { ctt tgc ctc ctc tct gaa act gg } \\
\text { R4 }\end{array}$ & 55 & 460 \\
F5 & $\begin{array}{l}\text { gat ct cga gct gac tga tgc } \\
\text { gaa gca gca atg ggc cag g }\end{array}$ & 55 & 386 \\
R5 & cat gat gag gaa gtg atg gta gc & & \\
R6 & &
\end{tabular}


Table 2a. Characteristics of primers used for CPT II gene PCR and sequencing

\begin{tabular}{|c|c|c|c|}
\hline Primer name & Sequence $\left(5^{\prime} \rightarrow 3^{\prime}\right)$ & $\mathrm{T}_{\text {an }}\left({ }^{\circ} \mathrm{C}\right)$ & Fragment length (bp) \\
\hline E1F & ctt gtg ttt aga ctc cag aac tcc c & 58 & 292 \\
\hline E1R & gtc atg agt gac tgc agt cag gtt g & & \\
\hline E2F & ctt gta aag cta att aac ctc ttc cat a & 52 & 192 \\
\hline E2R & tct tga acc acc cca act atg ctc & & \\
\hline E3F & cat gta ttc cct acc atg gtt tg & 58 & 277 \\
\hline E3R & cgt tac ttc att tgc tgg tct cac c & & \\
\hline $\mathrm{E} 4 \mathrm{~F}$ & ggg aca gca tta aca ttt tat gtt at & 60 & 1426 \\
\hline E4R & gta gaa tga ttt agg ctt gct tac cc & & \\
\hline E5F & ttt cet gag gtc ctt ttc cat cet g & 58 & 425 \\
\hline E5R & atg agg aag tga tgg tag ctt ttc a & & \\
\hline
\end{tabular}

Table 2b. Characteristics of internal primers used for CPT II exon 4 sequencing

\begin{tabular}{ll}
\hline Primer name & Sequence $\left(5^{\prime} \rightarrow 3^{\prime}\right)$ \\
\hline E4F2 & cgc ttt gtg cct tcc tct ctg \\
E4F3 & gat gag tag tgg caa tga gga gag c \\
E4F4 & caa ctt cga gct gac tga tgc \\
E4R1 & cga gtt gag ttg aaa agc cg \\
E4R2 & cag cat att gtg gga caa gtg g \\
E4R3 & ctt tgc ctc ctc tct gaa act gg \\
\hline
\end{tabular}

Genomic DNA analysis

Genomic DNA was isolated from cultured skin fibroblasts using the salt extraction method (Miller et al. 1988). PCR amplification of all five human CPT II exons with flanking intronic regions was performed according to the protocol for Taq polymerase (Life Technologies), by the use of the primers mentioned in Table 2a. All oligonucleotides were designed according to the human CPT II gene sequence published in GenBank (Verderio et al. 1995) (accession numbers U09642-U09646). PCR reactions were performed on $100 \mathrm{ng}$ DNA in $25 \mu \mathrm{l}$ total sample volume containing $50 \mathrm{ng}$ of each primer, $0.25 \mathrm{mM}$ dNTPs, $1.5 \mathrm{mM} \mathrm{MgCl}, 0.5$ units Taq polymerase, and PCR buffer (Life Technologies). PCR fragments were visualized by electrophoresis on a $1 \%$ agarose gel. Direct sequencing of the PCR products was performed using ABI PRISM Dye Terminators on the automated ABI PRISM 377 sequencer according to the manufacturer's protocol (Applied Biosystems). The PCR primers listed in Table 2a were also used for sequencing. Because of the length of exon 4, six internal primers were designed to sequence the complete fragment (Table $2 b$ ).

Segregation of both mutations within the family

From the available family members, a part of exon 4 (encompassing bp534) was amplified by PCR using the primers E4F (Table 2a) and E4R1 (Table 2b). PCR products were visualized by electrophoresis on a $4 \%$ agarose gel.

From the available family members, a genomic DNA fragment of exon 3 was amplified by PCR as described before. Because the splice-site mutation creates an MseI restriction site (TTAA), the PCR product was digested with MseI $(277 \mathrm{bp} \rightarrow 231 \mathrm{bp}+46 \mathrm{bp}$ when the mutation is present), and fragments were separated by gel electrophoresis on a $4 \%$ agarose gel.

Prenatal diagnosis

DNA was isolated from chorionic villi of three new pregnancies using the salt extraction method (Miller et al. 1988). Maternal contamination was ruled out using the AmpFLSTR Profiler Plus PCR amplification kit according to the manufacturer's protocol (Applied Biosystems). The presence of the 534Tins/del 25 mutation as well as the splice-site mutation was checked as described above.

\section{Results}

\section{Biochemical methods}

Measurement of CPT II activity was performed in cultured skin fibroblasts of the index patient. The specific CPT II activity was $1.1 \mathrm{nmol} /(\mathrm{mg}$ protein $\cdot \mathrm{min})$ (controls: $53.1 \pm$ 14.0), which amounts to about $2 \%$ of the mean control value.

\section{Mutational analyses}

\section{$m R N A$ analysis}

The complete open reading frame of the CPT II mRNA from both the index patient and controls was amplified in six overlapping fragments by PCR and visualized by agarose-gel electrophoresis. In the case of the patient's fragment 2, a single PCR fragment was obtained that appeared to be smaller than the wild-type fragment (Fig. 1a). Direct sequencing of all six PCR products revealed a single mutation in our index patient: a 534T insertion followed by a 25-bp deletion (encompassing bases 534-558), as published before (Yang et al. 1998). Because no wild-type fragment was amplified from the patient's cDNA, the mutation was characterized as homozygous. 
M

Co

IP

BI

M

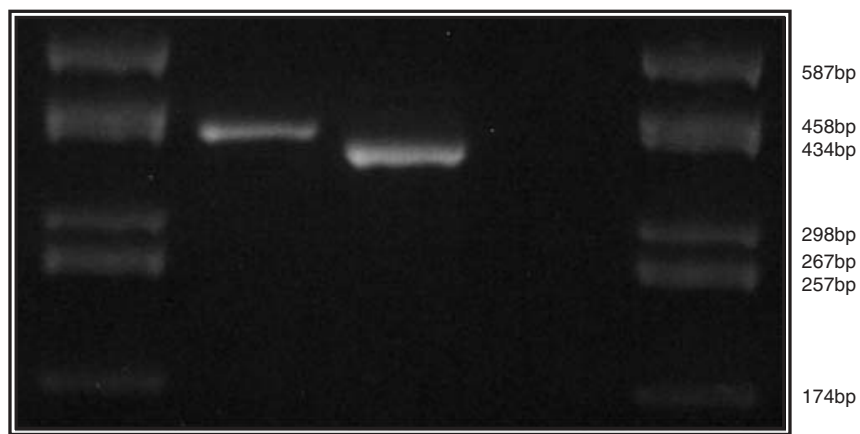

A
M
Co
IP
BI
M

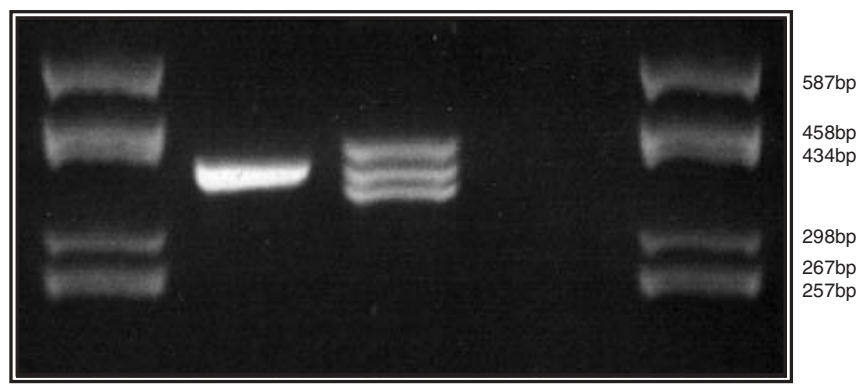

B

Fig. 1. A Carnitine palmitoyltransferase II (CPT II) cDNA fragments, amplified with polymerase chain reaction (PCR) primers F2 and R2, from a control $(\mathrm{Co})$ and the index patient $(I P)$, visualized on a $4 \%$ agarose gel. The control shows a single fragment of the expected length (405 bp), while the patient shows a single fragment that is smaller than the wild type. This suggests that presence of a homozygous deletion in the patient's cDNA. The pUC*HindIII molecular length marker $(M)$ and a no-template control (B1) are also shown. B CPT II genomic DNA fragments, amplified with PCR primers E4F and E4R1, from a control $(C o)$ and the index patient $(I P)$, visualized on a $4 \%$ agarose gel. The control shows a single fragment of the expected length ( $381 \mathrm{bp})$, while the patient shows three fragments: one equal to the wild type, one smaller fragment containing the deletion, and one longer fragment formed by both heteroduplexes. This proves the deletion to be heterozygous in the patient's cDNA. The pUC*HindIII molecular length marker $(M)$ and a no-template control $(B 1)$ are also shown

\section{Genomic DNA analysis}

To confirm the mutation at the genomic DNA level, a part of exon 4 of the CPT II gene was amplified by PCR and visualized by agarose-gel electrophoresis. Strikingly, three bands of different length appeared to be amplified: one equal to the wild-type fragment, one smaller band encompassing the deletion, and one longer fragment formed by both heteroduplexes (Fig. 1b). This proves the 534T ins/del 25 mutation to be heterozygous, which suggests the existence of a second mutation on the other allele.

Subsequently, all exons of the CPT II gene were amplified by PCR and visualized by agarose-gel electrophoresis. Direct sequencing of the fragments revealed the index patient to be compound heterozygous for the 534T ins/del 25 mutation in the exon 4 fragment, and a $\mathrm{g} \rightarrow \mathrm{a}$ splice-site mutation in the last nucleotide of intron 2 (Fig. 2). This new splice-site mutation was not detected among 100 control alleles.
atgttttagGAAAACAGA

Position of $\mathrm{g} \rightarrow \mathrm{a}$ mutation
Fig. 2. The splice-site mutation is located at the natural splice-acceptor site of intron 2 (in lowercase) and exon 3 (in uppercase), destroying the splice-recognition site

Segregation of both mutations within the family

Visualization of both mutations in the available family members reveals the 534Tins/del 25 mutation to have been inherited from the (heterozygous) father and the splice-site mutation to have been inherited from the (heterozygous) mother. All affected sibs appeared to be compound heterozygous for both mutations, while the healthy sib is heterozygous for only the splice-site mutation (Fig. 3).

\section{Prenatal diagnosis}

After maternal contamination was ruled out, chorionic villi DNA of three new pregnancies was checked for both mutations. This revealed the first to be heterozygous for the 534Tins/del 25 mutation, the second to be heterozygous for the splice-site mutation, and the third to be wild type (Fig. $3)$. These three pregnancies were continued uneventfully, and all children appeared to be healthy.

\section{Discussion}

Carnitine palmitoyltransferase II (CPT II) deficiency is an autosomal recessive disorder of mitochondrial fatty acid $\beta$ oxidation and presents as three clinically distinct phenotypes: onset and death in the first weeks of life (neonatal form), initial symptoms occurring later in infancy (infantile form), and adult onset (adult form). While several mutations have been related to the late-onset and infantile forms (Taroni et al. 1993), until now, only four individual mutations, all located in the CPT II gene, have been identified in individual patients suffering the neonatal form of CPT II deficiency (Bonnefont et al. 1999).

Mutation detection studies using cDNA in a CPT IIdeficient family implied that the index patient, the fourth affected child, was homozygous for a 534T insertion followed by a 25-bp deletion (encompassing bases 534-558), as published before (Yang et al. 1998). Studies on genomic DNA, however, revealed the patient to be compound heterozygous for the 534Tins/del 25 mutation and for a $\mathrm{g} \rightarrow \mathrm{a}$ splice-site mutation in the last nucleotide of intron 2 , which is the first splice-site mutation ever found in CPT II deficiency. It has been reported before that $\mathrm{g} \rightarrow \mathrm{a}$ transitions in the last position of an intron can affect splicing, either by exon skipping or by activating cryptic splice sites, resulting 


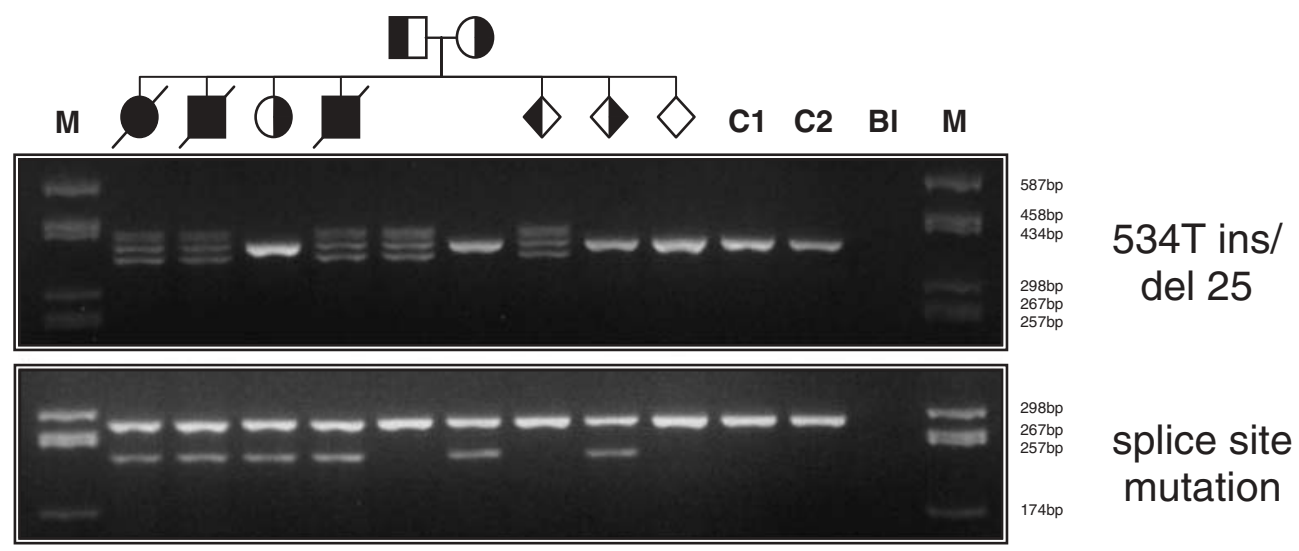

Fig. 3. PCR fragments encompassing both mutations were synthesized from all available family members and from chorionic villi (from which maternal contamination was ruled out) of three new pregnancies. The exon 4 fragment (534T ins/del 25 mutation), amplified with PCR primers E4F and E4R1 (381 bp), was visualized on a 4\% agarose gel. The exon 3 fragment (splice-site mutation), amplified with PCR primers E3F and E3R (277 bp), was digested by the MseI restriction enzyme, which cuts the mutated PCR product into a $230 \mathrm{bp}$ and a (not visible) $47 \mathrm{bp}$ fragment. Fragments were subsequently visualized on a $4 \%$ aga-

in an unstable mRNA transcript. Because PCR amplification with all possible cDNA primer pairs encompassing the exon 2-exon 3 boundary only resulted in the fragment containing the 534T ins/del 25 mutation (no abnormal-sized cDNA fragment could be detected), it can be concluded that the patient's splice-site mutation leads to mRNA degradation, proving its pathogenity. This study demonstrates the incompleteness of mutation detection at the mRNA level in the case of a mutation that results in an unstable mRNA transcript.

Segregation within the family shows that the father is heterozygous for the 534T ins/del 25 mutation, the mother is heterozygous for the splice-site mutation, and all affected children were compound heterozygous for both mutations. The nonaffected child carries only the splice-site mutation. Prenatal diagnosis performed in chorionic villi of three new pregnancies revealed the first to be heterozygous for the 534Tins/del 25 mutation, the second to be heterozygous for the splice-site mutation, and the third to have a wild-type genotype. These three children showed none of the symptoms that occurred in their deceased siblings.

Several pathogenic DNA mutations in the CPT II open reading frame have been identified in all three phenotypes. The distinct clinical phenotypes of CPT II deficiencies correlate in some extent to distinct genotypes. Truncating $C P T$ II mutations on both alleles are associated with the severe neonatal form of the disease, while homozygous and/or compound heterozygous "mild" missense mutations are often associated with the adult form of CPT II deficiency (Bonnefont et al. 1999). In the case where a patient is compound heterozygous for a severe and a mild CPT II mutation, the data show that the presence of the mild one is often sufficient to expose the patient to a mild clinical phenotype (Taggart et al. 1999; Thuillier et al. 2000; Yang et al. 1998). In our patients, the compound heterozygosity of the two rose gel. This revealed the father to be heterozygous for the 534T ins/ del 25 mutation and the mother and the healthy sib to be heterozygous for the splice-site mutation, whereas all affected sibs were clearly compound heterozygous for both mutations. Chorionic villi DNA of three new pregnancies revealed the first to be heterozygous for the $534 \mathrm{~T}$ ins/del 25 mutation, the second to be heterozygous for the splicesite mutation, and the third to be wild type. Two controls ( $C 1$ and $C 2)$, the pUC*HindIII molecular length marker $(M)$, and a no-template control (B1) are also shown

mutations led to the severe neonatal form of CPT II deficiency, so we can conclude that both mutations are severe. This proves that the new splice-site mutation leads to neonatal CPT II deficiency.

Acknowledgments We thank the patients and their family for participation, and Anouk Hanstede and Marloes Siers for technical assistance.

\section{References}

Bieber LL (1988) Carnitine. Annu Rev Biochem 57:261-283

Bonnefont JP, Demaugre F, Prip-Buus C, Saudubray JM, Brivet M, Abadi N, Thuillier L (1999) Carnitine palmitoyltransferase deficiencies. Mol Genet Metab 68:424-440

Chomczynski P, Sacchi N (1987) Single-step method of RNA isolation by acid guanidinium thiocyanate-phenol-chloroform extraction. Anal Biochem 162:156-159

Demaugre F, Bonnefont JP, Colonna M, Cepanec C, Leroux JP, Saudubray JM (1991) Infantile form of carnitine palmitoyltransferase II deficiency with hepatomuscular symptoms and sudden death. Physiopathological approach to carnitine palmitoyltransferase II deficiencies. J Clin Invest 87:859-864

DiMauro S, DiMauro PM (1973) Muscle carnitine palmitoyltransferase deficiency and myoglobinuria. Science 182:929-931

Fraser F, Corstorphine CG, Zammit VA (1997) Topology of carnitine palmitoyltransferase $\mathrm{I}$ in the mitochondrial outer membrane. Biochem J 323:711-718

Guzman M, Geelen MJ (1993) Regulation of fatty acid oxidation in mammalian liver. Biochim Biophys Acta 1167:227-241

McGarry JD, Woeltje KF, Kuwajima M, Foster DW (1989) Regulation of ketogenesis and the renaissance of carnitine palmitoyltransferase. Diabetes Metab Rev 5:271-284

Miller SA, Dykes DD, Polesky HF (1988) A simple salting out procedure for extracting DNA from human nucleated cells. Nucleic Acids Res 16:1215

Taggart RT, Smail D, Apolito C, Vladutiu GD (1999) Novel mutations associated with carnitine palmitoyltransferase II deficiency. Hum Mutat 13:210-220 
Taroni F, Verderio E, Fiorucci S, Cavadini P, Finocchiaro G, Uziel G, Lamantea E, Gellera C, DiDonato S (1992) Molecular characterization of inherited carnitine palmitoyltransferase II deficiency. Proc Natl Acad Sci USA 89:8429-8433

Taroni F, Verderio E, Dworzak F, Willems PJ, Cavadini P, DiDonato S (1993) Identification of a common mutation in the carnitine palmitoyltransferase II gene in familial recurrent myoglobinuria patients. Nat Genet 4:314-320

Thuillier L, Sevin C, Demaugre F, Brivet M, Rabier D, Droin V, Aupetit J, Abadi N, Kamoun P, Saudubray JM, Bonnefont JP (2000) Genotype/phenotype correlation in carnitine palmitoyl transferase
II deficiency: lessons from a compound heterozygous patient. Neuromuscul Disord 10:200-205

Verderio E, Cavadini P, Montermini L, Wang H, Lamantea E, Finocchiaro G, DiDonato S, Gellera C, Taroni F (1995) Carnitine palmitoyltransferase II deficiency: structure of the gene and characterization of two novel disease-causing mutations. Hum Mol Genet 4:19-29

Yang BZ, Ding JH, Dewese T, Roe D, He G, Wilkinson J, Day DW, Demaugre F, Rabier D, Brivet M, Roe C (1998) Identification of four novel mutations in patients with carnitine palmitoyltransferase II (CPT II) deficiency. Mol Genet Metab 64:229-236 